\title{
Characterization of AVHRR global cloud detection sensitivity based on CALIPSO-CALIOP cloud optical thickness information: demonstration of results based on the CM SAF CLARA-A2 climate data record
}

\author{
Karl-Göran Karlsson and Nina Håkansson \\ Swedish Meteorological and Hydrological Institute, Folkborgsvägen 17, 60176 Norrköping, Sweden \\ Correspondence: Karl-Göran Karlsson (karl-goran.karlsson@smhi.se)
}

Received: 22 August 2017 - Discussion started: 15 September 2017

Revised: 19 December 2017 - Accepted: 28 December 2017 - Published: 1 February 2018

\begin{abstract}
The sensitivity in detecting thin clouds of the cloud screening method being used in the CM SAF cloud, albedo and surface radiation data set from AVHRR data (CLARA-A2) cloud climate data record (CDR) has been evaluated using cloud information from the Cloud-Aerosol Lidar with Orthogonal Polarization (CALIOP) onboard the CALIPSO satellite. The sensitivity, including its global variation, has been studied based on collocations of Advanced Very High Resolution Radiometer (AVHRR) and CALIOP measurements over a 10-year period (2006-2015). The cloud detection sensitivity has been defined as the minimum cloud optical thickness for which $50 \%$ of clouds could be detected, with the global average sensitivity estimated to be 0.225 . After using this value to reduce the CALIOP cloud mask (i.e. clouds with optical thickness below this threshold were interpreted as cloud-free cases), cloudiness results were found to be basically unbiased over most of the globe except over the polar regions where a considerable underestimation of cloudiness could be seen during the polar winter. The overall probability of detecting clouds in the polar winter could be as low as $50 \%$ over the highest and coldest parts of Greenland and Antarctica, showing that a large fraction of optically thick clouds also remains undetected here. The study included an in-depth analysis of the probability of detecting a cloud as a function of the vertically integrated cloud optical thickness as well as of the cloud's geographical position. Best results were achieved over oceanic surfaces at mid- to high latitudes where at least $50 \%$ of all clouds with an optical thickness down to a value of 0.075 were detected. Corresponding cloud detection sensitivities over land surfaces
\end{abstract}

outside of the polar regions were generally larger than 0.2 with maximum values of approximately 0.5 over the Sahara and the Arabian Peninsula. For polar land surfaces the values were close to 1 or higher with maximum values of 4.5 for the parts with the highest altitudes over Greenland and Antarctica. It is suggested to quantify the detection performance of other CDRs in terms of a sensitivity threshold of cloud optical thickness, which can be estimated using active lidar observations. Validation results are proposed to be used in Cloud Feedback Model Intercomparison Project (CFMIP) Observation Simulation Package (COSP) simulators for cloud detection characterization of various cloud CDRs from passive imagery.

\section{Introduction}

Monitoring the global amount, distribution and optical properties of clouds is increasingly important because of the increasing evidence that the parametrization of cloud processes and cloud-aerosol interactions including related climate feedbacks are critical contributors to the uncertainty in climate change analysis and in predictions from climate models (Stocker et al., 2013). However, it is encouraging in this respect to note the steadily increasing amount of observations from spaceborne passive and active sensors (an excellent overview is available at https://www.wmo-sat.info/ oscar/) and the prolonged growth of the observational records from the initial satellite sensors launched in the 1970s. These 
early satellite observations, which consist of spectral radiance measurements, can be used to retrieve information about clouds and other relevant Earth-atmosphere parameters. Most importantly they have now evolved into time series of observations with lengths approaching 4 decades, which qualifies them for use as climate data records (CDRs). Examples of CDRs built upon such observations are described by Rossow and Schiffer (1999), Karlsson et al. (2017), Heidinger et al. (2014) and Stengel et al. (2017).

The advantage of using satellite-based observations for climate analysis is their global coverage. A similar coverage is very difficult to achieve with surface-based observations alone because of the sparsity of the surface-based observational network. This is particularly true for observations of cloudiness and cloud properties, where large parts of the Earth, especially oceanic and polar regions, are still poorly covered. The different observation capabilities and conditions for space-based sensors and surface-based observations also lead to problems when trying to characterize the accuracy of space-based CDRs. Although the quality of observations may be estimated for selected Earth positions or for small regions with dense surface networks, it is very difficult to achieve a representative and homogenous view of the accuracy over the entire globe using surface observations. The quality of CDRs is especially important as observations used for climate monitoring must be very accurate for the reliable estimation of potential climate change signals (Ohring et al., 2005), a central aspect in the planning and definition of the global climate observing system (Dowell et al., 2013). For this reason, there is also a need to become more stringent in the description of the uncertainty of CDRs by following international metrological norms (Merchant et al., 2017).

One solution for achieving both global coverage and improved quality description is to make use of high-quality reference measurements from spaceborne platforms (Dowell et al., 2013). This has already been successfully demonstrated by utilizing data delivered by the A-Train satellites, i.e. Afternoon Satellite Constellation, sometimes referred to as the Afternoon Train). This is a system of satellites operating in the same orbit configuration with nearly simultaneous observation times (Stephens et al., 2002). The most important satellite in the A-Train for the detection of clouds is the CALIPSO satellite, which has the Cloud-Aerosol Lidar with Orthogonal Polarization (CALIOP) onboard (Winker et al., 2009). The sensitivity of CALIOP to clouds in the atmosphere is much higher than for other space-based sensors and this makes it a natural reference for evaluating the cloud detection efficiency in data records compiled from passive sensor data (e.g. as demonstrated by Heidinger et al., 2016).

This paper presents a detailed CALIOP-based evaluation of the cloud detection efficiency and the uncertainty of the cloudiness information provided by CLARA-A2 (CM SAF cloud, albedo and surface radiation data set from AVHRR data - second edition) CDR (Karlsson et al., 2017). This CDR was released in 2017 by the Climate Monitoring Satel- lite Application Facility (CM SAF), a project belonging to the satellite ground segment of the European Organisation for the Exploitation of Meteorological Satellites (EUMETSAT; Schulz et al., 2009). The evaluation presented is based on an original validation method described by Karlsson and Johansson (2013) which has been extended with several new features. The method was first updated to use the latest revision of the CALIPSO-CALIOP data set (version 4) and results showing the impact of this change are presented. The study then takes advantage of the greatly extended CALIOP observation period (here covering almost 10 years) to monitor globally averaged cloud conditions in unprecedented detail. The achieved validation results, which cover approximately one-third of the CLARA-A2 observation period, can be considered to be the best currently available characterization of the global quality of the CLARA-A2 cloud data record. A specific enhancement of the original validation method is the estimation of the geographical distribution of cloud detection probability as a function of cloud layer optical thickness. Section 2 describes the CLARA-A2 and CALIPSO data sets, Sect. 3 outlines the extended validation method and the compiled validation data set and is followed by results in Sect. 4. Section 5 discusses the results and Sect. 6 provides conclusions and proposes potential future applications.

\section{Data}

\subsection{The CLARA-A2 climate data record.}

CLARA-A2 is constructed from historic measurements of the Advanced Very High Resolution Radiometer (AVHRR) operated onboard polar-orbiting NOAA satellites and the Metop polar orbiters operated by EUMETSAT since 2006. AVHRR measures radiation in five spectral channels (two visible and three infrared channels) with an original horizontal field-of-view (FOV) size at nadir of $1.1 \mathrm{~km}$. The data used in CLARA-A2 are a resampled version of these measurements at a reduced resolution of $5 \mathrm{~km}$, defined as global area coverage (GAC). The size is defined in this context as the approximate diameter (assuming a circular or elliptic shape) of the FOV and this definition will be used throughout this paper. Only resampled GAC data are available (i.e. being archived) globally over the full period since the introduction of the AVHRR sensor in space. The resampling of original data into GAC representation means that four out of five original FOVs are selected for the first scan line, while the next two scan lines are ignored. Radiances for these four selected FOVs are then averaged and used to represent the GAC FOV consisting of 15 original full-resolution FOVs. Thus, only about $25 \%$ of the nominal GAC FOV is actually observed (see also visualization in Fig. 1 in Sect. 3.2).

CLARA-A2 improves and extends the first version of the data record released in 2012 (Karlsson et al., 2013) and now 
covers a 34-year time period (1982-2015). Original visible radiances were inter-calibrated and homogenised, using MODIS (Moderate Resolution Imaging Spectroradiometer) data as a reference, before generating each component of the CLARA-A2 product portfolio. The inter-calibration was based on the method introduced by Heidinger et al. (2010), which has now been updated (MODIS Collection 6) and extended (6 years have been added). This updated calibration is described by Devasthale et al. (2017). CLARA-A2 features the following cloud products: cloud mask/cloud amount, cloud top temperature/pressure/height, cloud thermodynamic phase, cloud optical thickness (for liquid and ice clouds separately), particle effective radius and cloud water path. These cloud products are available as monthly and daily averages in a $0.25^{\circ}$ by $0.25^{\circ}$ latitude-longitude grid and also as daily resampled global products (Level $2 \mathrm{~b}$ ) in a $0.05^{\circ}$ by $0.05^{\circ}$ latitude-longitude grid. The daily resampled products are valid for each satellite and orbit node (ascending or descending), while the daily average product is an average of all available daily resampled products and the monthly products are the averages of all the daily average products. Cloud parameter results are also presented as multi-parameter distributions (i.e. joint frequency histograms of cloud optical thickness, cloud top pressure and cloud phase) for daytime conditions. In addition to cloud products, CLARA-A2 also includes surface radiation budget and surface albedo products and examples of the CLARA-A2 products can be found in Karlsson et al. (2017).

In this study, we focus exclusively on the quality of the original AVHRR GAC cloud mask because of its central importance to the quality of all other CLARA-A2 products. Validation results for other CLARA-A2 products can be found in Karlsson et al. (2017) and in CM SAF 1 (2017). The CLARA-A2 cloud mask is generated using an improved and extended version of the method first proposed by Dybbroe et al. (2005) which enables reliable processing of the historic AVHRR GAC record. These improvements are described in detail in Karlsson et al. (2017) and in CM SAF 2 (2017).

\subsection{The CALIPSO-CALIOP cloud information.}

An extensive description of the existing CALIPSO-CALIOP cloud and aerosol data sets can be found in Vaughan et al. (2009). In short, the Cloud Layer product from CALIOP (denoted CLAY) used in this study provides information on up to 10 individual vertically displaced cloud layers. As the detection of a cloud layer requires that all overlying layers are optically thin enough to allow the lidar signal to penetrate down to that particular layer, there can be a bias in the number of layers observed if overlaying clouds are optically thick. The CLAY product is provided in three different horizontal resolutions (along track): $333 \mathrm{~m}$ ("single shot"), 1 and $5 \mathrm{~km}$. The resolutions coarser than $333 \mathrm{~m}$ are constructed through averaging several single shots. This is done to increase the signal-to-noise ratio to allow detection of thinner clouds than could be achieved at the original single-shot resolution. Thus, CALIOP products at coarser resolution will be capable of detecting more clouds than at finer resolutions and it is preferable that studies of thin Cirrus clouds should be based on products in the coarsest resolution $5 \mathrm{~km}$ (Vaughan et al., 2009). Note that the nominal single-shot FOV size does not correspond to the true lidar FOV size but rather to the along-track sampling distance. As the true lidar FOV size is only $70 \mathrm{~m}$ (Winker et al., 2007), less than $5 \%$ of the nominal single-shot FOV is actually observed (see also Fig. 1 in Sect. 3.2).

An estimation of the cloud optical thickness of each layer is also provided but only for a FOV size of $5 \mathrm{~km}$. However these values are only reliable for clouds with relatively low optical thickness (below approximately 3), because of signal saturation in optically thick clouds (Vaughan et al., 2009; Sassen and Cho, 1992). In this study we have used the optical thickness interval $0-5$ because the new CALIPSO CLAY data set version 4.10 provides slightly increased cloud optical thickness values compared to previous versions. We interpret this change to represent underestimation in previous values. Despite this change there is still a high degree of uncertainty in values near the upper end of these limits and these may, in reality, include some clouds which are optically thicker.

The CALIPSO satellite follows the A-Train track in a sunsynchronous orbit with an Equator-crossing local time of 01:30. This means that observations from the NOAA satellites can be matched to CALIPSO-CALIOP data in nearnadir conditions for a full orbit if they are in an orbit with the same or very close to the same Equator-crossing time. For all other NOAA satellite orbits (and also the Metop satellites), matchups are only possible at high latitudes close to $70^{\circ} \mathrm{N}$ and S. Since CALIPSO is operated in a slightly lower and faster orbit than the NOAA/Metop satellites (i.e. orbital period of CALIPSO is $98.5 \mathrm{~min}$ while NOAA/Metop period is $102 \mathrm{~min}$ ), close matchups in time are found with a recurrence of approximately 2 days.

In this study, we have used the fourth reprocessed version of the CALIOP CLAY data sets (version 4.10), which was released in 2016. The main features of this updated version are described at https://www-calipso.larc.nasa.gov/ resources/calipso_users_guide/qs/cal_lid_12_all_v4-10.php.

Regarding the basic CALIOP cloud mask, the most relevant changes affecting this study are

1. revised and improved basic cloud-aerosol discrimination method;

2. removal of misclassifications of aerosols and dust as clouds at certain locations at high latitudes (as discussed by Jin et al., 2014);

3. inclusion of information on single-shot cloud detection in the $5 \mathrm{~km}$ data set, the implications of which are discussed in Sect. 3.3. 


\section{Validation analysis methods and data sets}

\subsection{Some theoretical considerations about clouds}

Cloudiness is not an absolute well-defined quantity like other cloud properties or most other geophysical parameters. Firstly, it depends on the scale of interest, i.e. the areal extent over which cloud cover has to be calculated needs to be specified. Secondly, and perhaps more importantly, a definition of what is meant by a cloud is required to allow a subsequent quantitative use of the results. For example, how optically thin or thick should a cloud be to be called a cloud? This threshold is important when studying the cloud impact on components of the radiation budget (Charlson et al., 2007; Barja and Antuña, 2011). How to define clouds detected in satellite imagery is also related to the scale of individual clouds (Koren et al., 2008). The cloud definition aspect is often missing in studies describing various cloud data records. Typically, products and validation results are presented without any deeper discussion on for which clouds are the results really valid. A good example is found in comparison studies between satellite-derived and manual surface-observed cloudiness (e.g. Sun et al., 2015). Results from such studies are difficult to interpret because of the different observation geometries for the compared data sets and the lack of an objective and clear definition of the clouds being observed in either of the two data sets. Because of this ambiguity it has often been recommended to use parameters other than cloudiness or cloud cover (as mentioned in WMO 1, 2012) to instead describe the effect of clouds (e.g. "cloud albedo", "effective cloud cover" or "joint histograms of cloud top pressure and cloud optical thickness") in climate analysis and climate model evaluation studies. Nevertheless, the need for the geographical distribution of modelled clouds to be correct is still a crucial requirement (as pointed out in WMO 1, 2012), particularly when considering that parameters describing the effect of clouds are still critically dependent on how you define the underlying cloud or cloud mask. This calls for continued studies of cloud cover from both the observational and modelling perspective. We propose here that the access to high-quality reference cloud observations from CALIPSO-CALIOP may help us take a significant step forward regarding the use of a more strict quantitative definition of cloudiness. A detailed characterization of the clouds we observe can be made using CALIOP data. Thus, the ability to observe similar clouds in data records based on passive imagery can then be assessed, which will augment the usefulness of these data records. The following subsections outline a new approach which will enhance the value of results from such cloud validation studies.

\subsection{Basic CALIOP matching method and matching geometry}

The underlying method for matching the two cloud data sets is described in detail by Karlsson and Johansson (2013). However, because of its importance for the understanding of method extensions and the results achieved in this study, we repeat here the most important aspects:

1. Positions where the orbital tracks cross are identified for the orbits of the two data sets to be collocated.

2. If the time difference of the two observations at the crossing point is within a certain maximum time difference $T_{\mathrm{diffmax}}$, the observations at this position are denoted simultaneous nadir observations (SNOs). Only orbits with SNOs satisfying the maximum $T_{\text {diffmax }}$ criterion are selected for further collocation studies. A $T_{\text {diffmax }}$ value of $45 \mathrm{~s}$ has been used in this study. As a consequence of a slightly shorter orbital period for the CALIPSO satellite, collocations could then be made with an approximate 2-day repeat cycle.

3. For NOAA satellites flying in an afternoon orbit (which is similar or almost similar to the orbit of CALIPSO), it is also possible to compare observations before and after the SNO point since both satellites continue to observe the same points on Earth close in time. For example, when using a maximum observation time difference of 3 min, almost all observations during an entire orbit along the CALIPSO track can be inter-compared. Not all observations from the NOAA satellite afternoon orbits will be made in nadir conditions; but all will be relatively close to nadir (i.e. within $15^{\circ}$ ). The current study uses afternoon orbit data with an observation time difference to CALIOP of $3 \mathrm{~min}$ to ensure global coverage.

4. For NOAA and METOP satellites flying in a morning orbit, the orbital tracks will cross almost perpendicularly and SNOs will then only occur at high latitudes (near $70^{\circ} \mathrm{N}$ and $\mathrm{S}$ ). A consequence of this is that collocations can only be made over distances limited by the AVHRR swath width. Furthermore, all individual collocations will then have varying AVHRR viewing angles along the matched track. Matchups with morning satellite data are not included in this study because of the limited geographical coverage.

In order to better understand the effects of different sensor sampling conditions and the collocation geometry, Fig. 1 shows an idealized representation of CALIOP collocations with AVHRR GAC data for both afternoon and morning orbits. The figure is idealized in the sense that it shows the perfect collocation, i.e. a collocation where the centre positions of both GAC and CALIOP FOVs are perfectly matched. We repeat that the AVHRR GAC sampling means that four out of five original FOVs are selected for the first scan line (marked 
(a) AVHRR-CALIOP matching for afternoon orbits

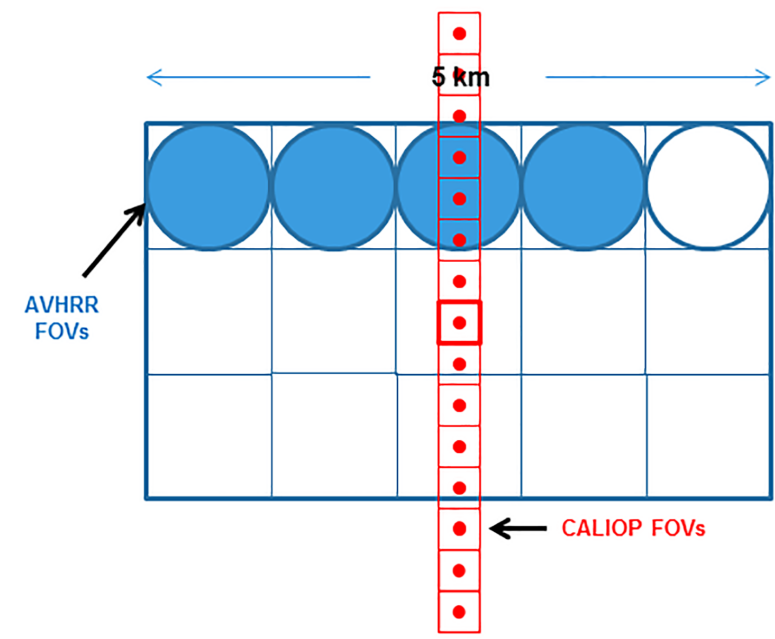

(b) AVHRR-CALIOP matching for morning orbits

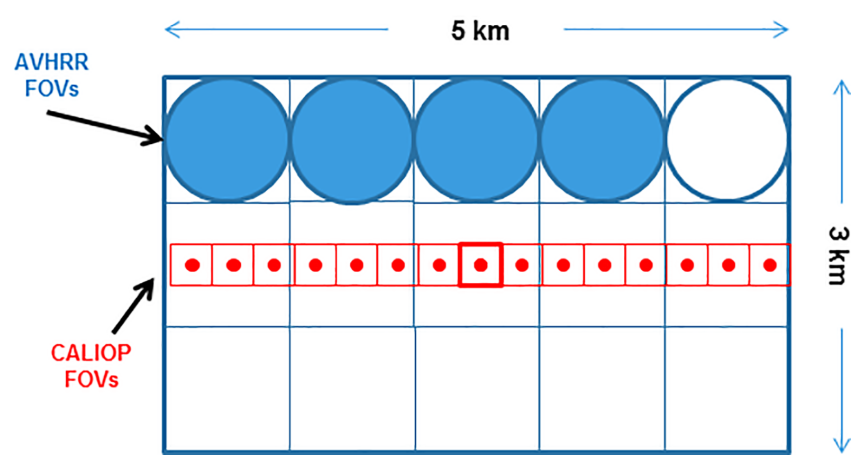

Figure 1. Matchup geometry for perfectly collocated AVHRR GAC and CALIOP FOVs for afternoon satellites (a) and morning satellites (b). The GAC FOV is visualized as a rectangle with sides of 3 and $5 \mathrm{~km}$ and with individual full-resolution AVHRR FOVs represented as $1 \mathrm{~km}$ squares. Blue circles indicate actual (more realistic) AVHRR measurements being used. Note that only the blue, filled AVHRR FOVs are averaged to represent the full GAC FOV. Red squares denote 15 original nominal 333 m CALIOP FOVs which represent the CALIOP $5 \mathrm{~km}$ FOV coverage. The highlighted centre FOV marks the position of the perfect match (i.e. at the centre of the GAC FOV). Note that the red, filled circles describe actual CALIOP measurements. See text for a more detailed explanation.

as a blue, filled circular FOVs in Fig. 1), while the next two scan lines are ignored (empty blue boxes in Fig. 1). Radiances for these four selected FOVs are averaged and then used to represent the entire GAC FOV consisting of 15 original full-resolution FOVs (schematically described as $3 \times 5$ blue boxes in Fig. 1). The $5 \mathrm{~km}$ CALIOP FOV observation is represented as an array of 15 original $333 \mathrm{~m}$ resolution red boxes in Fig. 1. Note that the true FOVs of the CALIOP sensor are smaller in size. In Fig. 1 they are represented as red, filled circles with $70 \mathrm{~m}$ size and separated by $333 \mathrm{~m}$. The $5 \mathrm{~km}$ CALIOP cloud observation is composed by averaging the 15 original measurements but also from averaging mea- surements outside of the nominal $5 \mathrm{~km}$ distance. This is done to detect optically very thin clouds (cirrus clouds) which could not be detected solely from data within the nominal $5 \mathrm{~km}$ FOV (as described by Vaughan et al., 2009).

The different panels for afternoon and morning orbit collocations in Fig. 1 are meant to illustrate how collocation conditions change from the along-track collocation mode for afternoon orbits to the across-track collocation mode for morning orbits. In contrast to afternoon satellites, the orbital tracks then crosses almost perpendicularly between CALIPSO and morning orbit satellites, explaining the shift to a horizontal instead of a vertical orientation of the array of CALIOP measurements in Fig. 1. The effects of the limited coverage of true AVHRR observations within the nominal GAC FOV and the different orientations of the array of CALIOP FOVs for morning and afternoon satellites can be ignored if cloud elements have scales larger than $5 \mathrm{~km}$. However, for cases with smaller scale (sub-pixel) cloud elements or cases with cloud edges within the GAC FOV, we can expect differences between AVHRR and CALIOP observations. The implications of this for the collocation and validation results will be discussed further in Sect. 5.

As explained by Karlsson and Johansson (2013), binary cloud masks for $5 \mathrm{~km}$ FOVs from AVHRR and CALIOP are inter-compared and evaluated using a range of standard validation scores. However, prior to comparison, the content of the original $5 \mathrm{~km}$ CALIOP FOV observation is adjusted to be consistent with the corresponding cloud mask defined at $1 \mathrm{~km}$ resolution. This check was introduced after noting that global CALIOP-estimated cloudiness for individual orbits was not always increasing when switching from the $1 \mathrm{~km}$ resolution data set to the $5 \mathrm{~km}$ resolution data set. Conceptually, cloudiness should increase for the $5 \mathrm{~km}$ data sets as it is better able to detect also the optically thinnest cloud layers in addition to those cloud layers detected at finer resolutions (Vaughan et al., 2009). However, a non-negligible fraction of cases $(\sim 3-5 \%$ of all investigated cases in a preparatory study) actually showed lower cloud amounts for the $5 \mathrm{~km}$ resolution. This inconsistency comes as a side effect of the actual method used for creating the coarser-resolution CALIOP data sets (Vaughan et al., 2009; David Winker, CALIPSO Science Team, personal communication, 2017). Prior to performing the horizontal averaging of the CALIOP scattering signal over several single shots, some single-shot views are excluded from the analysis if they contain strongly reflecting boundary layer clouds or aerosols. In the vast majority of cases, the number of these removed single shots is less than $50 \%$ of all single-shot measurements within the $5 \mathrm{~km}$ FOV. Considering the official $5 \mathrm{~km}$ FOV CALIOP cloud mask, this procedure would then still justify labelling the $5 \mathrm{~km}$ FOV as cloud-free if no other cloud layers are detected. However, in some areas the frequency of small-scale convective clouds may be high and for these cases this could lead to underestimated cloudiness in the $5 \mathrm{~km}$ products. Another important aspect is that strongly reflecting clouds on the sub-pixel 
scale of AVHRR GAC data may still be detectable because of non-linear radiance contributions (with similarities to the "hot spot" effect from fires) in the shortwave infrared channel at $3.7 \mu \mathrm{m}$ (Saunders and Grey, 1985; Saunders, 1986). Thus, to not include these clouds in the CALIOP data sets might lead to too low or non-representative validation scores for some of the investigated cases. Karlsson and Johansson (2013) showed that validation scores also improved for AVHRR-based cloud products when adding clouds from the $1 \mathrm{~km}$ data sets if three or more of the $1 \mathrm{~km}$ FOVs within the $5 \mathrm{~km}$ FOV were cloudy in cases when the original $5 \mathrm{~km}$ products were deemed cloud-free. For these added clouds from $1 \mathrm{~km}$ data, the $5 \mathrm{~km}$ cloud optical thickness (not estimated in CLAY $1 \mathrm{~km}$ data) was set to 5, i.e. at the maximum upper end of realistically estimated cloud optical thicknesses. This is a justifiable approach as these clouds are by definition strongly reflecting and in most cases would lead to effective cloud optical thicknesses close to or above 5 .

\subsection{Adaptation to CALIPSO version 4 CLAY products}

An important objective of this study was to verify that the method used by Karlsson and Johansson (2013) would still be applicable to the new version 4 of the CALIOP CLAY product released in 2016 and to investigate whether the validation results changed in any systematic way. Despite the modifications implemented (mentioned at the end of Sect. 2.2), the fundamental retrieval method for the CALIOP CLAY product has remained the same. Consequently, the abovementioned inconsistencies between fine- and coarseresolution CALIOP data sets are likely to remain and would need a similar post-processing adjustment as in previous version 3 products. However, the new version of the $5 \mathrm{~km}$ CALIOP cloud product (i.e. in this study we have used the standard CLAY product version 4.10) has been expanded to include full information on the single shots removed during the averaging process. Thus, the previous use of $1 \mathrm{~km}$ data in the method by Karlsson and Johansson (2013) could in principle be abandoned and replaced by the direct use of this single-shot removal information (the latter method to be called "modified method" in the following). Another improvement found in the version 4.10 data set is that the removed single-shot FOVs have also been labelled as being either cloudy or filled with thick aerosols. This separation was not available in version 3 where all removed single-shot FOVs were assumed to be cloudy. An inter-comparison of version 3 and version 4 products is presented in Sect. 4.1.

\subsection{Applied validation concept and validation scores}

Compared to the previous study by Karlsson and Johansson (2013) this study has access to CALIOP data for a much longer validation period; almost 10 years (2006-2015). This means that it is now possible to calculate the geographical distribution of validation results, in addition to global mean conditions. Due to a sufficiently large amount of AVHRRmatched nadir-looking CALIOP observations, for the first time it is possible to evaluate the quality of a cloud CDR in a (close to) homogeneous way over almost the entire globe with the only exception being close to the poles where CALIOP measurements are not available. Consequently, the validation results calculated in this paper are presented as global maps rather than as tables and figures with global mean values. For the plotting of these global maps the results have been rearranged and calculated using a Fibonacci grid with 28878 grid points evenly spread out around the Earth approximately $75 \mathrm{~km}$ apart. The resulting grid has almost equal area and almost equal shape of all grid cells making it preferable to traditional latitude-longitude grids which often introduce distortions near the poles. For further details on Fibonacci grids, see González (2009) and Swinbank and Purser (2006).

We have used the same set of validation scores as those described and defined by Karlsson and Johansson (2013), namely

- mean error (bias) of cloud amount (\%), describing the systematic error of the mean;

- bias-corrected root-mean-square error (RMSE) of cloud amount (\%), describing the random error of the mean;

- probability of detection $(0 \leq \mathrm{POD} \leq 1)$ for both cloudy and cloud-free conditions relative to all observed cloudy or clear cases;

- false alarm rate $(0 \leq \mathrm{FAR}) \leq 1)$ for both cloudy and cloud-free conditions relative to all predicted cloudy and clear cases;

- hit rate: frequency (value between 0 and 1) of correct cloudy and clear predictions relative to all cases;

- Kuipers skill score $(-1 \leq \mathrm{KSS} \leq 1)$ where value 1 means perfect agreement, value 0 means uncorrelated (random) results and value -1 means consistently opposite results (see Karlsson and Johansson for the exact definition).

The results are computed by treating both CLARA-A2 and CALIOP cloud masks as binary values - i.e. each FOV is considered as either fully cloudy or cloud-free. The Kuipers skill score can be used to better identify cases of misclassifications when one of the categories is dominating. The KSS is sensitive to misclassifications even if they occur in only a small minority of the studied cases. The KSS score aims to answer the question of how well the estimation separated cloudy events from cloud-free events.

A minimum requirement for describing the accuracy of a parameter is to estimate the mean error or bias (giving the systematic error) and the variance of the error (giving the random error or dispersion; Merchant et al., 2017). However, to enable the identification of specific problems with 
cloud identification it is necessary to look at the additional scores mentioned above, particularly in cases when one of the two categories ("cloudy" or "clear") is dominant. This is driven by the fact that any cloud contamination (even if it is just a few cases) can have serious implications for parameter retrievals further downstream in the processing. Therefore multiple validation scores are needed to correctly identify all problematic and critical cases.

\subsection{Extension of the original validation method: enhanced analysis and introduction of cloud layer detection probability}

The use of the CALIOP cloud mask for validation of cloud masking methods based on passive imagery is rewarding but also challenging. It is known from previous results which used the original CALIOP cloud mask that there is a large difference in sensitivity between CALIOP (high sensitivity) and passive sensors (moderate to low sensitivity) which leads to the question: how can this sensitivity difference be managed to ensure a generation of useful results?

There are two major risks when comparing cloud masks retrieved from passive sensors to the original CALIOP cloud mask:

1. The CALIOP data set will include sub-visible clouds (Martins et al., 2011) which are not possible to detect in passive imagery.

2. In areas where sub-visible clouds exist in abundance, a method may have been "overtrained" or "overfitted" (e.g. if trained with CALIOP data by statistical regression methods) to always predict clouds since this gives the best overall validation scores.

These two problems can be handled by focusing on what happens for clouds that have different vertically integrated optical thicknesses as provided by the CALIOP $5 \mathrm{~km}$ cloud product. By applying successively reduced CALIOP cloud masks in the validation exercise we may exclude the thinnest clouds from the analysis by transforming them into cloudfree FOVs. This also means that we can isolate clouds within finite cloud optical thickness intervals (i.e. by subtracting two adjacent restricted CALIOP cloud masks with different filtered cloud optical thicknesses) in order to calculate validation results exclusively for this subset of clouds. If the cloud optical thickness interval is sufficiently small and the number of samples within each interval is sufficiently high we may then estimate the method's efficiency in detecting a cloud (i.e. the cloud layer detection probability $\operatorname{POD}_{\text {cloudy }}(\tau)$, where $\tau$ is the mean optical thickness or depth in the given interval) with this particular cloud optical thickness. We may then expect to see low detection scores for small optical thicknesses with scores improving as cloud optical thickness values increase. We argue that a special situation occurs when this cloud layer detection probability exceeds $50 \%$ for the first time for increasing cloud optical thicknesses. This marks an important performance point which could be seen as a minimum performance requirement: at this cloud optical thickness we detect at least $50 \%$ of all clouds. In the following we will denote this value of the filtered cloud optical thickness as the method's cloud detection sensitivity. There should also be a peak in the hit rate parameter at exactly this point. For small optical thicknesses, scores would improve if we filter out thin clouds, while for larger optical thicknesses, scores start to decrease as too many correctly detected clouds are transformed into the cloud-free case. We maintain that the best way to evaluate a cloud masking method is to estimate this cloud sensitivity parameter and to re-compute all validation scores after applying optical thickness filtering using exactly this value. This describes a method's optimal performance when using CALIOP cloud masks as the reference. The cloud detection sensitivity parameter defines the method's cloud detection capability in terms of the thinnest cloud that can confidently be detected. Furthermore, the validation scores computed at this value of the filtered optical thickness then define the method's optimal performance (in terms of the hit rate) taking into account also false classifications. An important complementary parameter in this context is the false alarm rate in the unfiltered case $\left(\operatorname{FAR}_{\text {cloudy }}(\tau=0)\right)$ since this parameter does not depend on any filtering of optically thin clouds. $\operatorname{FAR}_{\text {cloudy }}(\tau=0)$ can be used to investigate the degree of overtraining of a method (according to second bullet above). In the following Sect. 4, we present results of the cloud detection sensitivity and a range of validation scores computed at the point of the cloud detection sensitivity (i.e. using a CALIOP cloud mask filtered for thin clouds using the cloud detection sensitivity parameter as the optical thickness threshold). Most of these results are presented as global maps.

\subsection{The final compiled validation data set}

We have matched a total of 5747 global afternoon orbits of the NOAA-18 and NOAA-19 satellites with corresponding CALIPSO-CALIOP data in the time period October 2006 to December 2015. Due to increasing orbital drift of the NOAA-18 satellite after 2010 (with resulting deviation from the A-Train orbit and increasing off-nadir viewing angles for matchups), the matchup data set contains a small fraction of observations with higher satellite zenith angles. The observation time difference is limited to $3 \mathrm{~min}$ and the spatial matchup error was maximized to $2.5 \mathrm{~km}$ (as a consequence of using the nearest-neighbour technique and after assuming negligible geolocation errors). This resulted in more than 23 million global matchups. The distribution of the matchups is shown in Fig. 2 using a Fibonacci grid resolution of $75 \mathrm{~km}$.

Figure 2 shows a large variation in coverage as a function of latitude with a minimum number of matchups occurring at low latitudes and a maximum of matchups for the high- 


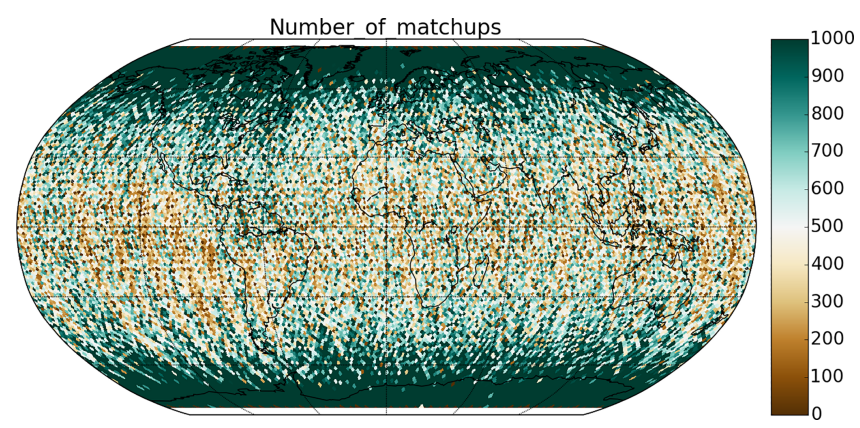

Figure 2. Total number of CALIPSO-CALIOP matchups with NOAA-18 and NOAA-19 AVHRR observations in the time period October 2006 to December 2015. Results are presented in a Fibonacci grid with $75 \mathrm{~km}$ resolution.

est latitudes. Although the likelihood for a valid matchup to occur is the same everywhere on a particular matched orbit, the pattern of the matchup numbers is explained by the converging orbital tracks towards the poles. Furthermore, the large variation with some distinct features (e.g. over the Pacific Ocean) shows that it was not possible to extract all theoretically available matching cases (some periods with loss of data exist for both CALIOP and AVHRR). Although it does not have fully homogeneous global coverage, the data set represents the best possible effort for full coverage that we can make at present. Even at low latitudes the number of matches generally exceeds 300 for a grid resolution of $75 \mathrm{~km}$, with only a few exceptions mainly located over the Pacific Ocean. In these locations the uncertainty in the results might be expected to be larger than for the rest of the globe.

\section{Results}

\subsection{Results from inter-comparisons of validation results based on CALIPSO-CALIOP version 3 vs. version 4}

Results from the modified validation method were compared against results from the old method for a test data set of 80 NOAA-18 CALIPSO-matched orbits between October and December 2006. These results are presented in Figs. 3 and 4. Figure 3 shows validation results for the two different approaches based exclusively on CALIOP CLAY version 4.10 products. The visualization used here, showing the results for two validation scores (hit rate and Kuipers score; see also discussion and definition in Sect. 3.4) is identical to the approach seen in Karlsson and Johansson (2013). Results using the original CALIOP cloud mask are given by the leftmost value with a filtered cloud optical thickness of 0.0 . The curves represent validations which use a successively reduced CALIOP cloud mask where clouds optically thinner than the values on the $x$ axis have been transformed from cloudy to clear cases. In this way we can calculate for which

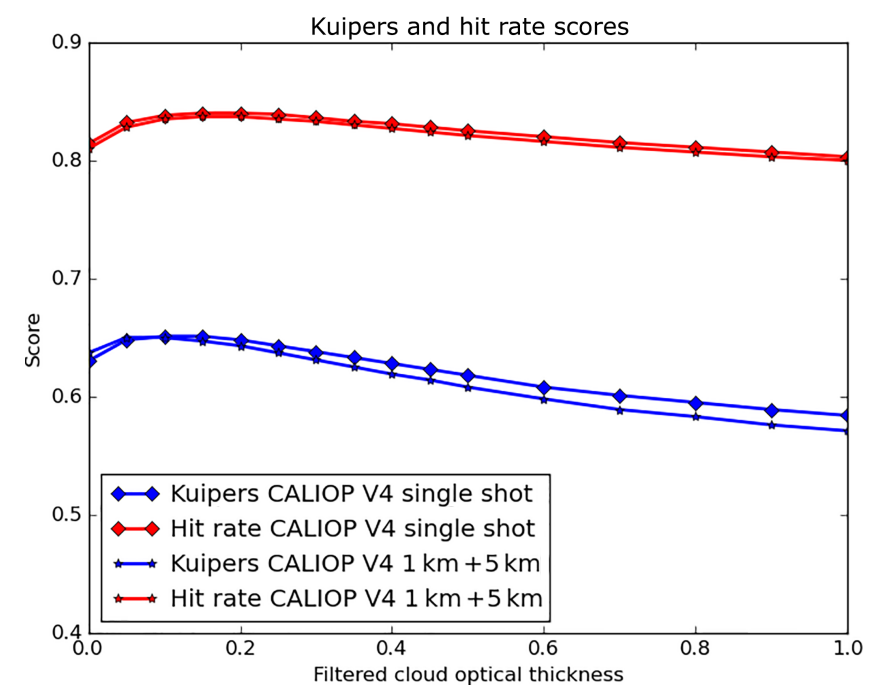

Figure 3. CALIOP-based validation scores (hit rate and Kuipers) as a function of filtered cloud optical thickness (see text for explanation) for 80 matched NOAA-18 orbits between October and December 2006. Validation is based on CALIOP version 4.10 CLAY products and show results from two alternative validation methods (single shot or combined 1 and $5 \mathrm{~km}$; see text for explanation).

CALIOP cloud mask (i.e. for which filtered cloud optical thickness) we get the highest scores. Figure 3 shows slightly improved results for the method using the single-shot information, although they are practically identical. The slight improvement may be attributed to the improved cloud-aerosol labelling of removed single shots. Figure 4 shows the overall effect of introducing the new matching method and the new version 4 data set compared to the results achieved using the former version 3 data set and the previous matching method. There is a small increase in the overall results (maximum scores) and a progression of the maximum values towards larger optical depths. The improvement in results indicates an improved CALIOP product, and the shifting of peak score values towards larger filtered cloud optical depths is indicative of more realistic and larger optical depths in CALIOP version 4.10 data (as confirmed by David Winker, CALIPSO Science Team, personal communication, 2017). These results are in line with expectations and demonstrate that the modified method is an appropriate basis for further validation studies based on the updated CALIOP CLAY data set.

\subsection{Results based on original CALIOP cloud masks compared to results excluding contributions from very thin clouds}

Figure 5 shows the global distribution of the hit rate parameter when comparing to the original CALIOP cloud mask. Results indicate fairly good cloud screening capability over mid- to high latitudes (especially over oceans) but degraded 


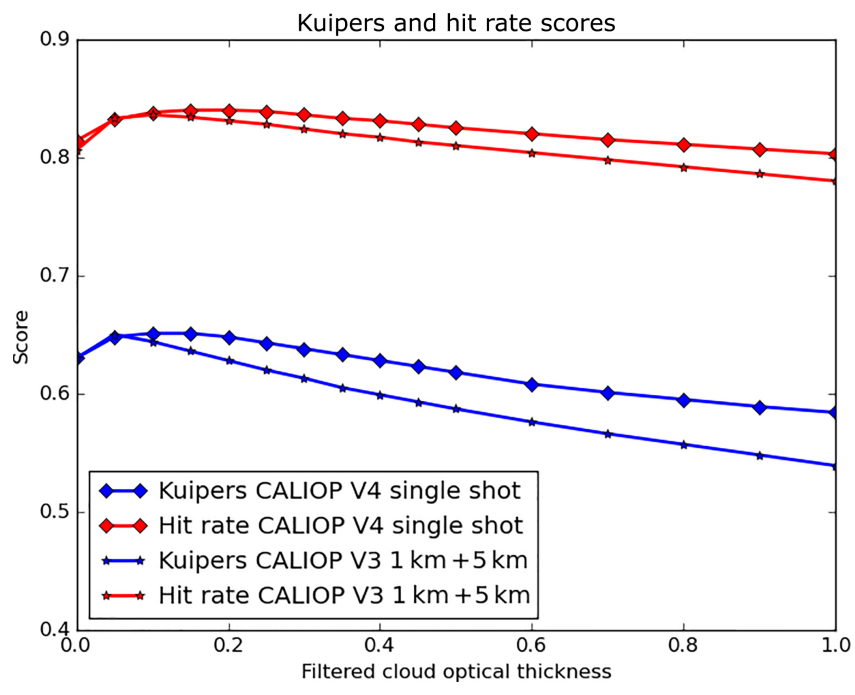

Figure 4. CALIOP-based validation scores (hit rate and Kuipers) as a function of filtered cloud optical thickness (see text for explanation) for 80 matched NOAA-18 orbits between October and December 2006. The curves compare results based on CALIOP version 4.10 CLAY products computed with the new method based on single-shot information (denoted "CALIOP V4 single shot") with results based on CALIOP version 3.01 CLAY products computed with the old method based on combined 1 and $5 \mathrm{~km}$ data (denoted "CALIOP V3 1 and $5 \mathrm{~km}$ ").

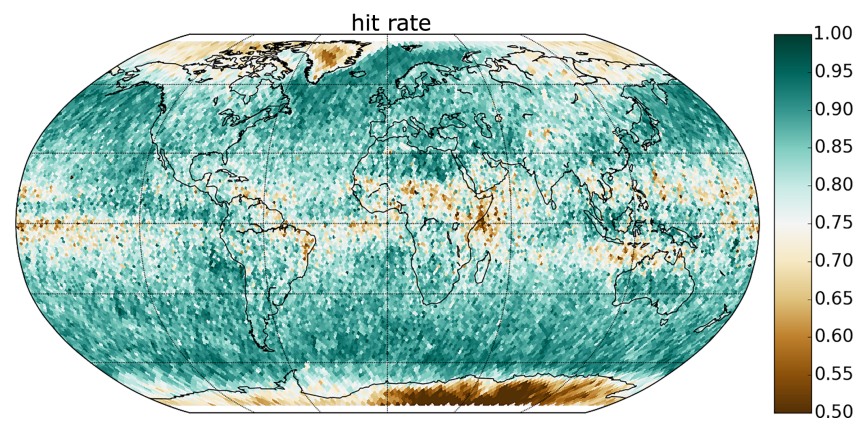

Figure 5. Global presentation of the CLARA-A2 cloud mask hit rate parameter with a horizontal Fibonacci grid resolution of $75 \mathrm{~km}$. Validation results are based on comparisons with the original CALIPSO-CALIOP cloud mask. Same underlying matchup data set as in Fig. 2.

results at most low latitudes and over the polar regions. The poorest results occur over Greenland and Antarctica.

Further analysis of results is complicated by the fact that the original CALIOP cloud mask includes all CALIOPdetected clouds as explained in Sect. 3.5. In particular, we suspect that the rather poor results in Fig. 5 in the tropical region may be significantly influenced by the presence of subvisible clouds.

By using all available matchups, we can calculate $\operatorname{POD}_{\text {cloudy }}(\tau)$ for all values of $\tau$ (Fig. 6) using the method outlined in Sect. 3.5. Calculations are based on optical thick-

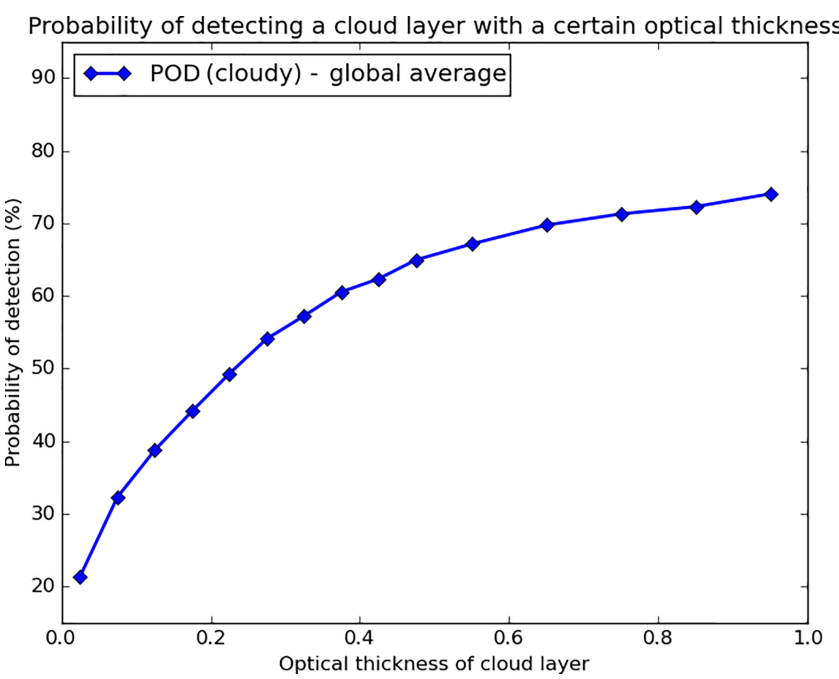

Figure 6. Global estimation of the probability of detecting a cloud with a certain cloud optical thickness. Calculations are based on all available AVHRR-CALIOP matchups over the time period October 2006 to December 2015.

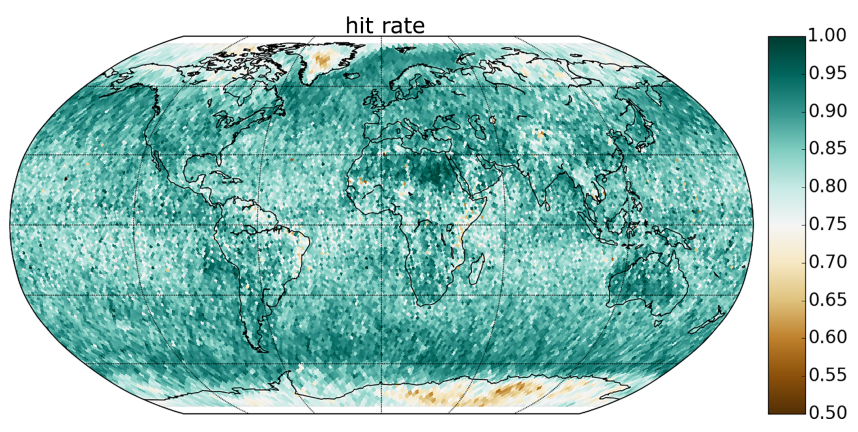

Figure 7. Peak hit rate results for the CLARA-A2 cloud mask achieved after filtering the CALIOP cloud mask with the cloud optical thickness value of 0.225 . Same underlying matchup data set as in Fig. 2. Results are presented in a Fibonacci grid with $75 \mathrm{~km}$ resolution.

ness intervals of 0.05 in the range $0.0<\tau<0.5$, intervals of 0.1 in the range $0.5<\tau<1.0$, and intervals of 1.0 in the range $1.0<\tau<5.0$ (results from the latter interval are not shown in Fig. 6). Figure 6 shows that the cloud detection sensitivity (i.e. where a probability of $50 \%$ is reached) is 0.225 for the investigated AVHRR-based results. Consequently, we will use this value to indicate the optimal hit rate results, with the global distribution of these results presented in Fig. 7. As expected, the results improve considerably for most locations compared to Fig. 5, especially over low latitudes. Hit rates above $80 \%$ are now achieved over most regions. The polar regions (at least the snow- and ice-covered parts) stand out as regions of poor quality with the worst results seen over central Greenland and Antarctica. There is also some degradation in the results over some regions at low-to-middle latitudes. 
The results in Fig. 7 give a much clearer measure of the cloud detection capability of the CLARA-A2 cloud screening method than those shown in Fig. 5, because they are now linked to a well-defined description of the involved clouds. We will apply the same filtering approach to obtain the results shown in the next subsection.

\subsection{Additional validation scores}

Figure 8 presents results for the systematic (bias) and random errors (bias-corrected RMSE) of the CLARA-A2 cloud amounts. It is clear that the cloud detection problems over the polar regions, as indicated by the hit rate parameter in Fig. 7, lead to a significant underestimation of cloud amounts, especially over those areas normally covered with snow or ice. However, this is an overall mean (close to an annual mean) and the underlying results may be seasonally varying. For example, cloud detection in the polar summer season is considerably better than during the polar winter (as shown by Fig. 6 in Karlsson et al., 2017). The results with least bias are found over mid- to high latitudes while some overestimation is seen over lower latitudes, particularly over oceanic surfaces. RMSE values are high in the polar regions and over what can be described as oceanic subtropical high regions. This agrees well with the corresponding hit rate results seen in Fig. 6. RMSE values are low over dry desert regions but mostly as a consequence of the general lack of cloudy situations here.

To further investigate areas where there is significant misclassification of cloudy and clear conditions we can study results of probability of detection of the cloudy and clear categories in Fig. 9. For the cloudy category, results are consistent with those deduced from previous figures with the exception of the low probabilities of cloud detection over northern Africa and the Arabian Peninsula. For the clear category we note high values over predominantly dry land portions of the world while low values are seen over the tropical region and over oceanic storm track regions at high latitudes.

Results for the Kuipers score are shown in Fig. 10. This score does not show as much regional variability as the hit rate score. Again, we note low score values over the snowcovered polar regions and over some desert regions. The largest difference to the hit rate is seen over high-latitude oceanic regions, where the Kuipers scores show rather modest values while hit rate showed relatively high score values.

Figure 11 shows the corresponding false alarm rates for cloudy and clear conditions. We note high false alarm rates for cloudy conditions over tropical and subtropical regions (with some dominance for oceanic regions) while for clear conditions the largest false alarm rates are found in the polar regions.
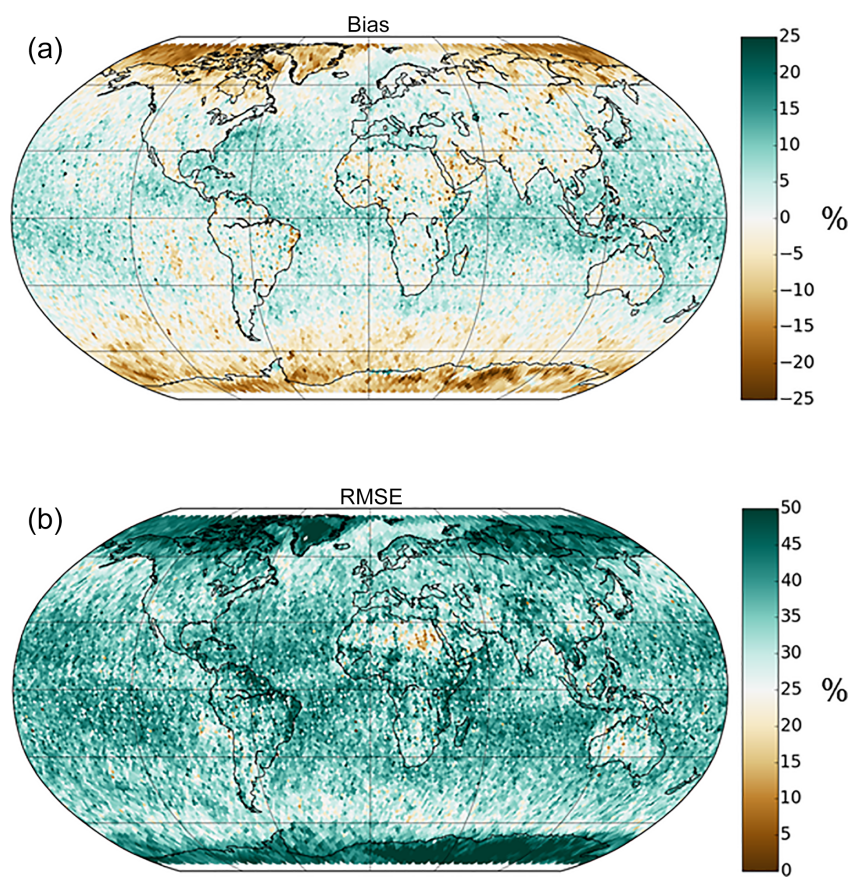

Figure 8. Mean error (bias) and bias-corrected root-mean-square error (RMSE) for the CLARA-A2 cloud amount achieved after filtering the CALIOP cloud mask with the cloud optical thickness value of 0.225 . Same underlying matchup data set as in Fig. 2. Results are presented in a Fibonacci grid with $75 \mathrm{~km}$ resolution.

\subsection{Estimating the global variability of cloud detection limitations}

Here we present presented validation results after having "removed" (in the sense of interpreting them as cloud-free cases) all clouds with smaller optical depths than the cloud detection sensitivity parameter. This leads to a clear improvement in the results when compared to the original CALIOP cloud mask (i.e. comparing Figs. 5 and 7). However, the cloud detection sensitivity value currently applied is a global average which could contribute to the large geographical variations in the results. To investigate how serious this simplification is, we can plot the results of $\tau_{\min }(\mathrm{POD}>50$ ) calculated exclusively for every Fibonacci grid point (Fig. 12). To reduce the uncertainty in this calculation due to low sample per grid point numbers as indicated in Fig. 2 for low latitudes, we have increased the radius of the Fibonacci grid from 75 to $300 \mathrm{~km}$. Figure 12 shows considerable variation in cloud detection sensitivity over the globe. It is clear that the cloud detection sensitivity is considerably lower than the global average value of 0.225 over most oceanic areas as well as over tropical land areas. On the other hand, values are generally larger than 0.225 over dry and desert-like regions and over high-latitude and polar land areas. For the polar land areas the cloud detection sensitivity frequently exceeds 1 and for some grid points even reaches values close to 5 . These 

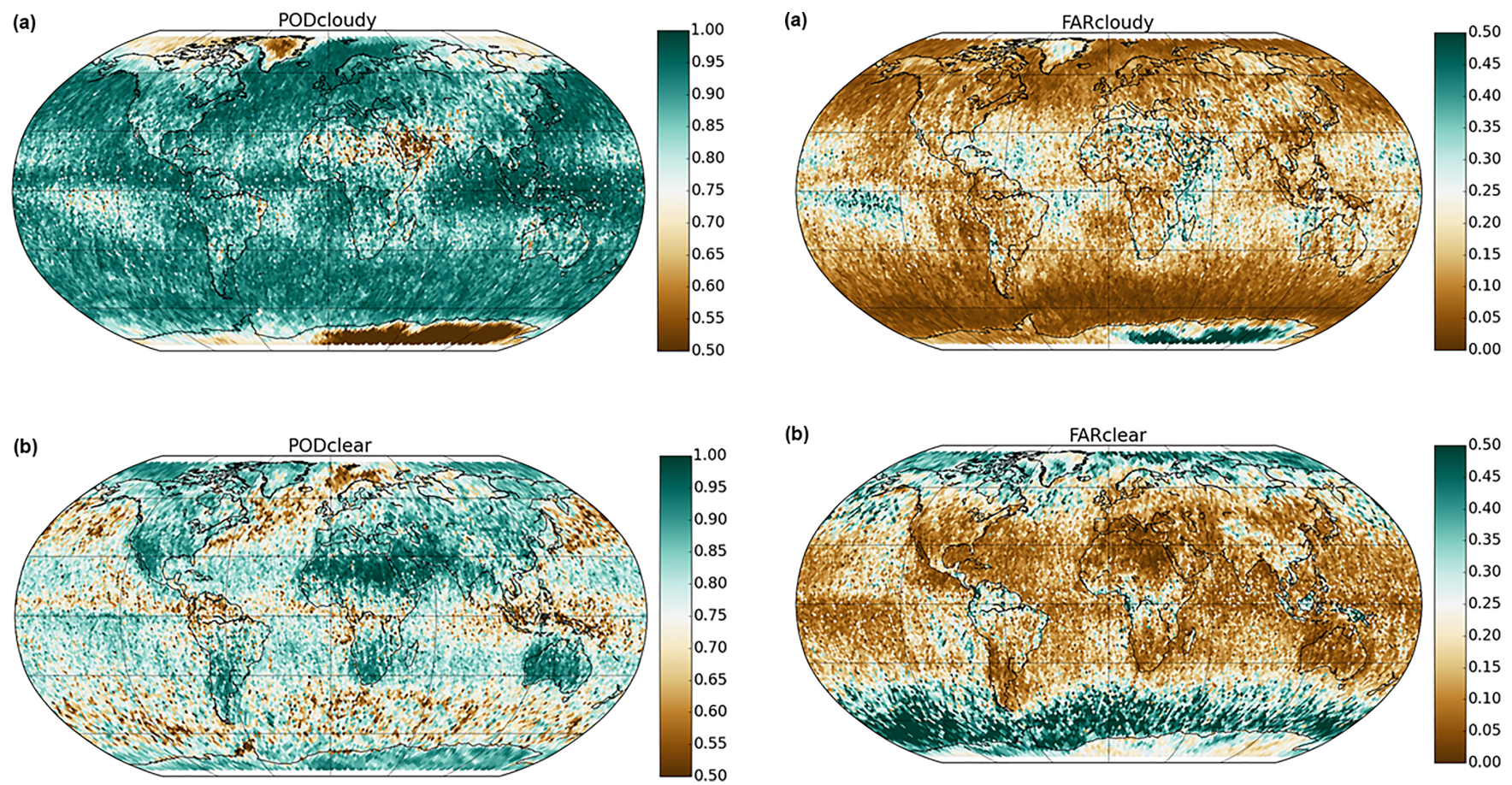

Figure 9. Probability of detection of cloudy (a) and clear (b) conditions for the CLARA-A2 cloud mask achieved after filtering the CALIOP cloud mask with the cloud optical thickness value of 0.225 . Same underlying matchup data set as in Fig. 2. Results are presented in a Fibonacci grid with $75 \mathrm{~km}$ resolution.

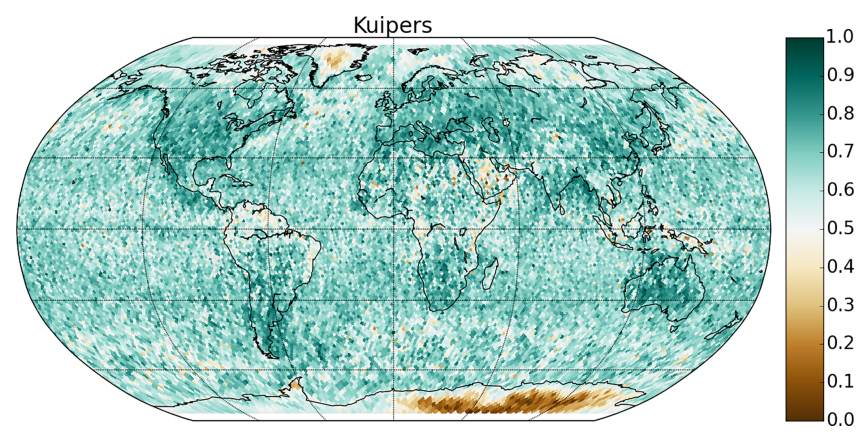

Figure 10. Kuipers score for the CLARA-A2 cloud mask achieved after filtering the CALIOP cloud mask with the cloud optical thickness value of 0.225. Same underlying matchup data set as in Fig. 2. Results are presented in a Fibonacci grid with $75 \mathrm{~km}$ resolution.

values contrast with the global average value of 0.225 , indicating that more representative (and most likely higher) validation scores could have been achieved if globally resolved cloud detection sensitivity values were used to re-calculate each of the validation scores. However, we have not taken this step here because of the relatively low number of samples in some grid points (even at the $300 \mathrm{~km}$ scale).

We can also visualize the variable cloud detection sensitivity by plotting the same kind of cloud layer probability curves as in Fig. 6 for a selection of individual grid points. Figure 13

Figure 11. False alarm rates for cloudy (a) and clear (b) predictions for the CLARA-A2 cloud mask achieved after filtering the CALIOP cloud mask with the cloud optical thickness value of 0.225. Same underlying matchup data set as in Fig. 2. Results are presented in a Fibonacci grid with $75 \mathrm{~km}$ resolution.

shows these curves for the three locations marked in Fig. 12. The blue curve in Fig. 13 shows cloud layer detection probabilities for a distant (from land) point in the North Atlantic Ocean. It marks a position where cloud detection is clearly most effective compared to the global average. The cloud detection sensitivity value is 0.075 at this location demonstrating that even very thin clouds are well detected there. The cloud detection capability also reaches a maximum value of approximately $95 \%$ by $\tau=0.5$. This is considered to be as high as can be reached because of the limitations of the data sets, which include, for instance, the remaining and unavoidable AVHRR-CALIOP mislocation and matching problems (both in time and space). In contrast, a grid point located in the Sahel region (green curve in Fig. 13) shows worse results with a cloud detection sensitivity of 0.375 and maximum cloud detection capability only observed at $\tau=3.5$ and higher. However, a more extreme case is the location over central Greenland (red curve in Fig. 13). The cloud detection sensitivity here is as large as 1.5 and even at a maximum $\tau$ value of 4.5 we cannot come close to achieving optimal cloud detection capability. Thus, over a snow-covered and often extremely cold location we cannot even detect all optically thick clouds, consistent with the low POD $_{\text {cloudy }}$ results seen over Greenland and Antarctica in Fig. 9a.

The results in Fig. 13 again indicate that the validation matchup data set slightly undersamples the true conditions 


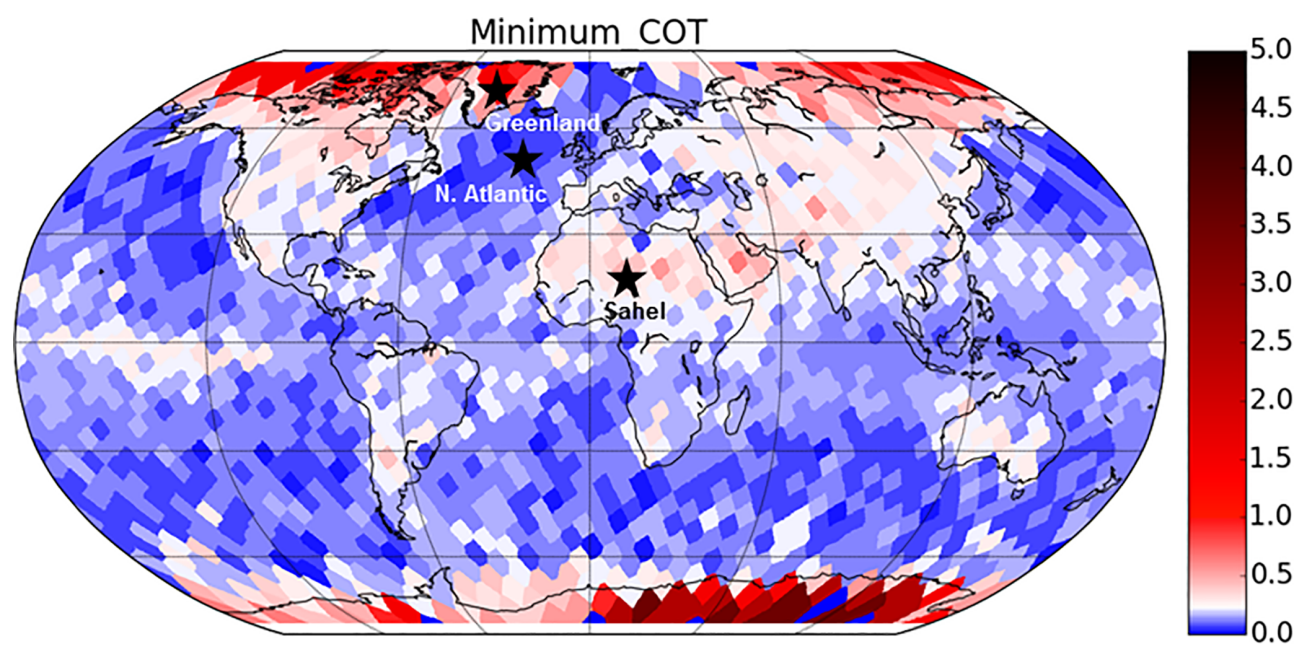

Figure 12. Global map of estimated cloud detection sensitivity of the cloud mask of CLARA-A2 (see text for explanation). Results are calculated from the same data set as visualized in Fig. 2 but in a coarser Fibonacci grid resolution of $300 \mathrm{~km}$. Conditions in the three marked locations (black stars) are analysed further in Fig. 13. Values below the global mean value of 0.225 are coloured in shades of blue and values above the global mean value are coloured in shades of red.

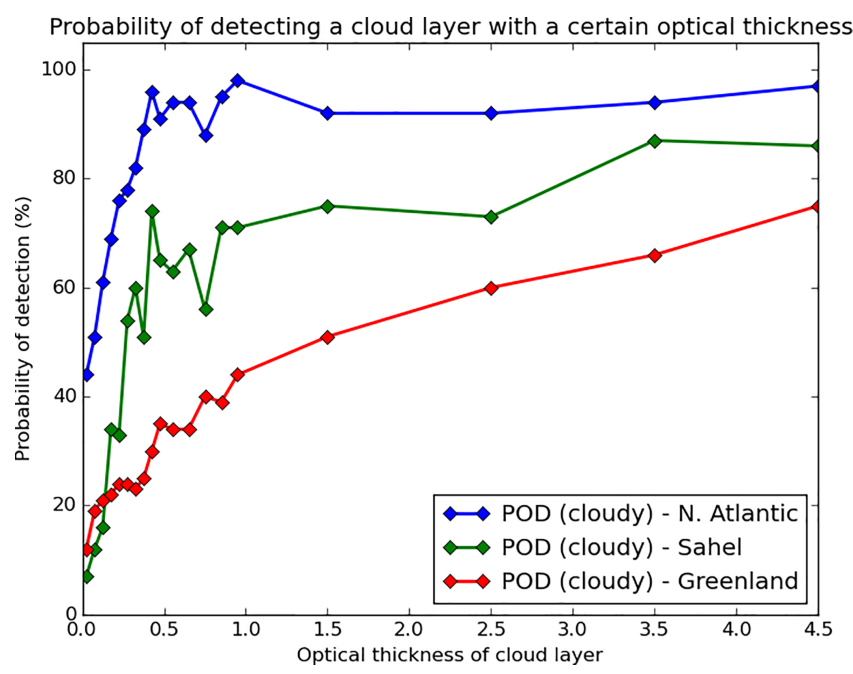

Figure 13. Same as Fig. 6 but for the individual grid points marked in Fig. 12.

for a limited number of grid points. This is indicated by the unexpected decrease in POD at some points for increasing $\tau$ values. Theoretically, one would expect a steady increase in POD as a function of $\tau$.

\section{Discussion}

There are several features of the results depicted in Figs. 711 which warrant further attention and discussion. One of these is the reduction in performance observed over areas which are known to be dry and mostly cloud-free. The POD $_{\text {cloudy }}$ results in Fig. 9 show particularly low values over the Sahara and the Arabian Peninsula. This indicates that in these particular areas, where cloudiness is generally low, CLARA-A2 still has difficulty detecting the few cloudy cases which occur. The exact reasons for this have to be investigated further but are likely linked to remaining uncertainties in the surface emissivities used over these semi-arid regions and deserts.

Another feature to discuss is the overestimation of cloudiness over low and middle latitudes (especially over oceans) seen in the bias plot in Fig. 8. This feature illustrates how it is difficult to find a simple representative way of evaluating results while also taking into account the existence of sub-visible clouds. The method applied in Fig. 8 (and in all Figs. 7-11) is to ignore cloud contributions in the CALIOP data set for clouds having an optical thickness less than 0.225. But, as already mentioned in Sect. 4.4, the latter value is a global mean value and in many places on Earth clouds with smaller optical thicknesses are actually detected confidently. This is clearly demonstrated in Fig. 12, where the cloud detection sensitivity over oceanic surfaces is noticeably better (smaller) than the global mean of 0.225 . This means that by applying the global value 0.225 as the filtering threshold of CALIOP-detected clouds, many clouds which were originally correctly detected in CLARA-A2 will now be treated as being falsely detected. If a locally representative value of the cloud detection sensitivity (as shown in Fig. 12) is used for the CALIOP filtering procedure, this apparent overestimation of clouds would largely disappear. However, to confidently apply such localized filtering a larger set of collocated observations is required to remove the sensitivity to low numbers of samples in individual grid points. Such a study will be possible in a few more years once an even larger matchup data set has been collected. An extended data 
set could also allow a further subdivision of the data set to study the diurnal and seasonal variation of the validation results.

A more interesting and general feature is shown in Fig. 9: in areas where cloudiness is low (e.g. over subtropical ocean and land regions) $\mathrm{POD}_{\text {cloudy }}$ is low, and where cloudiness is high (e.g. over mid-latitude storm tracks and near the Equator) $\mathrm{POD}_{\text {clear }}$ is low. This explains to a large extent the fairly low values of the Kuipers score over these regions (Fig. 10) leading to a slightly different distribution of results in comparison to the hit rate (Fig. 7). However, we must remember that hit rate is dominated by results for the dominating mode (cloudy or clear) while the Kuipers score highlights more clearly the existence of misclassifications of the minority mode. Figures 9 and 10 reveal that even if the dominantly cloudy and clear regions are generally captured very well the few cases of the opposing mode have a high frequency of misclassifications. This result is difficult to understand from the perspective of long-term experience of AVHRR cloud screening, as cloud screening works best over dark and warm ocean surfaces in good illumination. So, why are results not better here (e.g. over oceanic subtropical high regions)? We believe that this unexpected behaviour is a consequence of the limitations of both AVHRR GAC data and CALIPSOCALIOP data when it comes to the sampling of the true conditions within the nominal $5 \mathrm{~km}$ FOV.

To understand this we have to go back to Fig. 1 displaying the conditions for the matching of AVHRR GAC and CALIOP observations and the overall collocation geometry. Sections 2.1 and 3.2, together with Fig. 1, clearly describe how only about $25 \%$ of the nominal $5 \mathrm{~km}$ AVHRR GAC FOV is actually observed by AVHRR and that the corresponding figure for CALIOP single-shot nominal FOV of size $330 \mathrm{~m}$ is as low as $5 \%$. Note that the latter means that CALIOP is only able to cover about $0.3 \%$ of the nominal $5 \mathrm{~km}$ FOV. This has important consequences for all cases where we have cloud elements present which are smaller in size than the nominal $5 \mathrm{~km}$ FOV. We can first conclude that only in those cases containing cloud elements larger than the nominal $5 \mathrm{~km}$ FOV can we be confident that AVHRR and CALIOP observations will be comparable. For all other cloud situations involving clouds smaller than $5 \mathrm{~km}$ or when a cloud edge occurs within the GAC FOV, the two data sources will give different results since the sensors will observe different parts of the $5 \mathrm{~km}$ FOV. The situation is compounded by the fact that the AVHRR scan lines are perpendicular to the CALIPSO track when matching the two data sets in the near-nadir mode (Fig. 1a). This means that the CALIOP sensor consistently probes a different part of the nominal $5 \mathrm{~km}$ FOV to AVHRR. Theoretically, a maximum of three CALIOP single-shot measurements (out of a total of 15) would be able to measure the same spot on Earth as the AVHRR GAC measurement within the FOV size of $5 \mathrm{~km}$. However, it is clear from Fig. 1 that in a non-negligible fraction of cases, the two sensors will not even observe any common part of the nominal GAC FOV. This occurs when the nearest-neighbour matching of GAC and CALIOP FOVs places the CALIOP FOV in the rightmost part of the GAC FOV (see Fig. 1a). A direct consequence of these differences between the actual AVHRR and CALIOP measurements is that, in the case of dominating fractional cloudiness with cloud size modes below the $5 \mathrm{~km}$ scale, the random errors and the false alarm rates will increase even if the overall bias remains small (assuming that the cloud element distribution within the GAC FOV is random over a long time period, i.e. as expected for climate data records). This behaviour is exactly what is observed over the oceanic subtropical high regions (Figs. 8a and 11a) and also explains the degraded overall scores in this region (in particular the $\mathrm{POD}_{\text {cloudy }}$ score in Fig. 9) relative to other surrounding regions.

These regions of interest also have a reduced total cloud amount in the annual mean (e.g. see Fig. 6 in Karlsson et al., 2017), mainly because of the more stable atmospheric conditions there. The prevailing large-scale subsidence (poleward parts of the Hadley cell) in these locations suppresses cloudiness in mid- to high layers and is conducive only to the formation of convective and stratiform boundary layer clouds. This boundary layer cloudiness consists mainly of scattered small-scale cumulus and stratocumulus clouds, i.e. typically the kind of clouds for which we would expect enhanced disagreement between the AVHRR and CALIOP data sets as a result of variability within the $5 \mathrm{~km}$ FOV. It is interesting to note that this feature is not exclusive to oceanic areas. In addition, some eastern parts of continents show similar results, e.g. easternmost part of South America and Africa. This could indicate that scattered cumulus cloudiness is also the dominant mode of cloudiness in these locations. Finally, note also that we can see exactly the same effect for fractional clear areas, e.g. over Northern and Southern Hemisphere storm tracks at mid- to high latitudes as shown by the

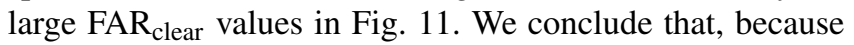
of the problems with correctly representing cases of both small-scale cloudiness and small-scale holes in cloud decks in the two data sets, the validation results could be underestimated (i.e. giving too low scores) over these dominantly cloudy or dominantly clear regions of the globe. This reduction of scores would then be largely attributed to mismatches due to GAC FOV geolocation errors (which are not zero), matchup errors (explained by the nearest-neighbour matching of GAC and CALIOP FOVs) and to the different cloud representation in each data set rather than to real cloud detection problems. Thus, examination of the cloud detection capability of a method should also take into account the scales of clouds being investigated. A consequence of this is that detailed studies of small-scale convective cloudiness should rather be based on original-resolution AVHRR and CALIOP observations than on data sets with a coarse-resolution data representation.

Finally, a specific problem with the applicability of the current method is the inability to assess the global quality 
of products from polar satellites in morning orbits (e.g. from the NOAA-17 and Metop satellites) as a consequence of CALIPSO following an afternoon orbit. Matchups with CALIPSO-CALIOP are consequently only possible at high latitudes leaving low-to-middle latitudes without reference observations for AVHRR products. Previous cloudiness comparisons for morning satellites at high latitudes (CM SAF 1, 2017) show good agreement with corresponding results from afternoon satellites (assuming that diurnal cycle cloud effects are small at high latitudes). Thus, for cloud amount information (in contrast to some other cloud parameters, like cloud effective radius) there is no reason to suspect large differences between morning and afternoon results even if morning orbit data partly use measurements in another spectral band (at $1.6 \mu \mathrm{m})$ in the shortwave infrared spectral region. However, this needs to be confirmed in the future through the use of reference data from the Cloud-Aerosol Transport System lidar (CATS, https://cats.gsfc.nasa.gov/) on the International Space Station or by use of data from the Earth Cloud Aerosol and Radiation Explorer (EarthCARE) mission (http://m.esa.int/Our_Activities/Observing_the_ Earth/The_Living_Planet_Programme/Earth_Explorers/ EarthCARE/ESA_s_cloud_aerosol_and_radiation_mission) for new afternoon satellites with two coexisting shortwave infrared channels onboard (e.g. NOAA-20).

\section{Conclusions}

We have shown that with access to the latest cloud information provided by the high-sensitivity CALIPSO-CALIOP lidar (CALIOP version 4.10 data set, covering almost a full decade, 2006-2015) it is possible to construct a detailed global analysis of the cloud detection sensitivity and other skill scores of the cloud screening method used in the AVHRR-based CLARA-A2 cloud climate data record. A wide range of validation scores, including those complementary to the essential scores describing systematic and random errors, have been used to obtain a very detailed picture of the cloud screening efficiency of CLARA-A2. Furthermore, by use of the CALIOP-derived information on cloud optical thickness, it has been possible to make a clear definition of which clouds have been observed and thus for which clouds the validation scores are valid. We believe this to be crucial to the further quantitative use of the results. The method is not specifically developed or valid exclusively for the CLARAA2 cloud masking method; it is also applicable to any method utilizing CALIOP data as a reference. Consequently, we propose that this method be used in future inter-comparisons of results from different cloud masking methods and cloud CDRs (following the example by Stubenrauch et al., 2013).

It is necessary to specify the clouds being investigated because the CALIOP sensor is capable of detecting clouds which are fundamentally "sub-visible" for passive imaging sensors. Therefore, a globally estimated minimum cloud op- tical thickness value (denoted "Cloud detection sensitivity"), for which the majority of clouds would be detected, was estimated to be 0.225 for the CLARA-A2 cloud masking method. This value was used to remove contributions to validation scores from thinner clouds than this minimum optical thickness, thus maximising the validation scores. For example, by utilising this definition of detectable clouds, resulting cloud amounts were found to be unbiased over most locations of the world except for a major underestimation over the polar regions. For the latter, a large part of all clouds still remains undetected during the polar night and this fraction can be as high as $50 \%$ over the coldest and highest portions of Greenland and Antarctica. Under these conditions not even optically thick clouds may be detected due to the very similar thermal characteristics of clouds and Earth surfaces. Landocean differences were generally small with only results over Greenland and Antarctica standing out as clear exceptions.

The study revealed some interesting reductions in performance over mainly subtropical ocean areas. In these locations random errors were elevated indicating a decrease in agreement between AVHRR and CALIOP observations despite otherwise very favourable cloud detection conditions (e.g. warm ocean temperatures and good illumination conditions). We argue that this is caused by the different sampling conditions within the studied $5 \mathrm{~km}$ FOV of the AVHRR and the CALIOP sensors, which is particularly evident in cases where small-scale boundary layer cloudiness dominates the cloud situation. Because of this we suspect that the cloud detection capability over these areas could actually be better than that shown by these results.

An important novel feature of this study compared to many previous validation efforts based on CALIPSO-CALIOP data is the estimation of the probability of detecting an individual cloud as a function of its vertically integrated optical thickness and its geographical position on Earth. This was accomplished by isolating finite optical thickness intervals in the CALIOP cloud information and calculating validation scores for this subset of data in a coarse global grid. Results show a substantial variation compared to the global mean optical thickness value of 0.225 for the thinnest retained cloud in the CALIOP cloud mask to give optimal global validation scores. The highest sensitivity to clouds in AVHRR data is generally found over mid- to high-latitude ocean surfaces. Here, clouds with cloud optical thicknesses as low as 0.075 can be detected efficiently. This is in comparison to a value of approximately 0.2 over tropical oceans and typically greater than 0.2 over most land surfaces. The latter value reaches 0.5 over some dry and desert-like regions (e.g. the Sahara and the Arabian Peninsula) and increases towards or beyond 1 over polar regions with the highest value of 4.5 found over Greenland and Antarctica. These results indicate that not even optically thick clouds can be confidently identified over Greenland and Antarctica during the polar winter. While these are not entirely new findings (e.g. see Karlsson and Dybbroe, 2010), this study has increased the confidence in the valida- 
tion results over the polar regions. Consequently, these results could help in optimizing the combined use of passive and active cloud observations over the polar areas in specific process and radiation studies (similar to earlier work by Kay and Gettelman, 2009; Kay and L'Ecuyer, 2013).

The presented validation method can be viewed as a step towards a more stringent and universal validation method to be used consistently for cloud climate data records generated from passive imagery (as discussed in Wu et al., 2017). The more than decade-long CALIPSO-CALIOP cloud data set should be used for benchmarking and for evaluation of current CDRs and future revisions of them. The method presented here could be seen as one candidate method. The ability to derive globally distributed results makes it easier to define and test global quality requirements for the CDRs. For example, requirements could be formulated in terms of minimum global coverage within a certain quality threshold instead of today's often overly generalized global requirement which uses one finite value or a value range (WMO 2, 2011).

One particular aim of this study was to provide a strict definition of the clouds being validated alongside the main validation results. This has been accomplished through the use of the CALIOP-derived cloud mask and the CALIOP-estimated optical thickness of clouds. As a result these validation results are more quantitatively useful. One obvious application would be to incorporate this information about strengths and limitations of cloud detection capabilities into the cloud data set simulators of the Cloud Feedback Model Intercomparison Project (CFMIP) Observation Simulation Package (COSP; Bodas-Salcedo et al., 2011). Existing COSP simulators for cloud data sets generated from passive satellite imagery (e.g. ISCCP and MODIS) do not explicitly take into account these potential inherent cloud detection problems and instead, they concentrate on simulating some satellite-specific or retrievalspecific features (e.g. systematic underestimation of cloud top height of thin high clouds), leaving it to the user of the simulator to add existing knowledge on cloud detection efficiency in the final evaluation process. It would clearly be beneficial if aspects of cloud detection capabilities were to be explicitly accounted for in these simulators. A specific CLARA-A2 COSP simulator is therefore under development where the description of such quality aspects will be included based on the findings of this validation study.

Finally, we repeat our opinion that CALIPSO-CALIOP data are an invaluable asset for the current and future evaluation of cloud CDRs based on passive satellite imagery. At the same time, we must express our concern about the current uncertainty regarding the long-term planning of possible replacements of both the A-Train satellites and the upcoming EarthCARE mission. Without follow-on missions it will be very difficult to assess the critical long-term stability of these CDRs, which in turn increases the difficulty in assessing the reliability of any climate trends deduced from these CDRs. There is also a need to slowly transform CLARAtype data records to AVHRR heritage data records, i.e. ex- tend the AVHRR results into the future using results from similar spectral channels from other sensors (e.g. the VIIRS sensor on recently launched and future polar NOAA satellites). Continued access to observations from active spaceborne lidar systems is essential for the development of such AVHRR heritage data records.

Data availability. CALIPSO-CALIOP data sets were obtained from the NASA Langley Research Center Atmospheric Science Data Center (ASDC DAAC - https://eosweb.larc.nasa.gov). The CLARA-A2 data record is (as all CM SAF CDRs) freely available via the website https://www.cmsaf.eu.

Author contributions. KGK conducted the validation study and wrote the manuscript. NH prepared the calculation and visualization of the global results.

Competing interests. The authors declare that they have no conflict of interest.

Acknowledgements. The authors want to thank David Winker in the CALIPSO Science Team for valuable advice regarding the use of CALIOP cloud information. The authors are also grateful to David Dufton for constructive comments on the manuscript.

This work was funded by EUMETSAT in cooperation with the national meteorological institutes of Germany, Sweden, Finland, the Netherlands, Belgium, Switzerland and the United Kingdom.

Edited by: James Campbell

Reviewed by: Hartwig Deneke and two anonymous referees

\section{References}

Barja, B. and Antuña, J. C.: The effect of optically thin cirrus clouds on solar radiation in Camagüey, Cuba, Atmos. Chem. Phys., 11, 8625-8634, https://doi.org/10.5194/acp-11-8625-2011, 2011.

Bodas-Salcedo, A., Webb, M. J., Bony, S., Chepfer, H., Dufresne, J.-L., Klein, S. A., Zhang, Y., Marchand, R., Haynes, J. M., Pincus, R., and John, V. O.: COSP: Satellite simulation software for model assessment, B. Am. Meteor. Soc., 2011, 1023-1043, https://doi.org/10.1175/2011BAMS2856.1, 2011.

Charlson, R. J., Ackermann, A. S., Bender, F. A.-M., Anderson, T. L., and Liu, Z.: On the climate forcing consequences of the albedo continuum between cloudy and clear air, Tellus, 59B, 715-727, https://doi.org/10.1111/j.16000889.2007.00297.x, 2007.

CM SAF 1: Validation Report - CM SAF Cloud, Albedo, Radiation data record, AVHRR-based, Edition 2 (CLARA-A2) Cloud Products, SAF/CM/DWD/VAL/GAC/CLD version 2.3, https://doi.org/10.5676/EUM_SAF_CM/CLARA_AVHRR/V002, 2017.

CM SAF 2: Algorithm Theoretical Basis Document - CM SAF Cloud, Albedo, Radiation data record, 
AVHRR-based, Edition 2 (CLARA-A2) - Cloud Fraction, SAF/CM/DWD/ATBD/CMA_AVHRR version 2.0, https://doi.org/10.5676/EUM_SAF_CM/CLARA_AVHRR/V002, 2017.

Devasthale, A., Raspaud, M., Schlundt, C., Hanschmann, T., Finkensieper, S., Dybbroe, A., Hörnquist, S., Håkansson, N., Stengel, M., and Karlsson, K.-G.: PyGAC: An open-source, community-driven Python interface to preprocess the nearly 40year AVHRR Global Area Coverage (GAC) data record, GSICS Quartherly Newsletter, Summer 2017, Special Issue on ReProcessing, 11, https://doi.org/10.7289/V5R78CFR, 2017.

Dowell, M., Lecomte, P., Husband, R., Schulz, J., Mohr, T., Tahara, Y., Eckman, R., Lindstrom, E., Wooldridge, C., Hilding, S., Bates, J., Ryan, B., Lafeuille, J., and Bojinski, S.: Strategy Towards an Architecture for Climate Monitoring from Space, 39 pp., available at: www.ceos.org; www.wmo.int/sat; http:// www.cgms-info.org/ (last access: 31 January 2018), 2013.

Dybbroe, A., Thoss, A., and Karlsson, K.-G.: NWCSAF AVHRR cloud detection and analysis using dynamic thresholds and radiative transfer modelling - Part I: Algorithm description, J. Appl. Meteorol., 44, 39-54, 2005.

González, A.: Measurement of Areas on a Sphere Using Fibonacci and Latitude-Longitude Lattices, Math. Geosci., 42, 49-64, https://doi.org/10.1007/s11004-009-9257-x, 2009.

Heidinger, A., Foster, M., Botambekov, D., Hiley, M., Walther, A., and Li, Y.: Using the NASA EOS A-Train to Probe the Performance of the NOAA PATMOS-x Cloud Fraction CDR, Remote Sens., 8, 511, https://doi.org/10.3390/rs8060511, 2016.

Heidinger, A. K., Straka, W. C., Molling, C. C., Sullivan, J. T., and Wu, X. Q.: Deriving an inter-sensor consistent calibration for the AVHRR solar reflectance data record, Int. J. Remote Sens., 31, 6493-6517, https://doi.org/10.1080/01431161.2010.496472, 2010.

Heidinger, A. K., Foster, M. J., Walther, A., and Zhao, Z.: The Pathfinder Atmospheres Extended (PATMOS-x) AVHRR climate data set, B. Am. Meteorol. Soc., 95, 909-922, 2014.

Jin, Y., Okamoto, J., and Hagihara, Y.: Improvement of CALIOP cloud masking algorithms for better estimation of dust extinction profiles, J. Meteorol. Soc. Jpn., 92, 433-455, https://doi.org/10.2151/jmsj.2014-502, 2014.

Karlsson, K.-G. and Dybbroe, A.: Evaluation of Arctic cloud products from the EUMETSAT Climate Monitoring Satellite Application Facility based on CALIPSO-CALIOP observations, Atmos. Chem. Phys., 10, 1789-1807, https://doi.org/10.5194/acp10-1789-2010, 2010.

Karlsson, K.-G. and Johansson, E.: On the optimal method for evaluating cloud products from passive satellite imagery using CALIPSO-CALIOP data: example investigating the CM SAF CLARA-A1 dataset, Atmos. Meas. Tech., 6, 1271-1286, https://doi.org/10.5194/amt-6-1271-2013, 2013.

Karlsson, K.-G., Riihelä, A., Müller, R., Meirink, J. F., Sedlar, J., Stengel, M., Lockhoff, M., Trentmann, J., Kaspar, F., Hollmann, R., and Wolters, E.: CLARA-A1: a cloud, albedo, and radiation dataset from $28 \mathrm{yr}$ of global AVHRR data, Atmos. Chem. Phys., 13, 5351-5367, https://doi.org/10.5194/acp-135351-2013, 2013.

Karlsson, K.-G., Anttila, K., Trentmann, J., Stengel, M., Fokke Meirink, J., Devasthale, A., Hanschmann, T., Kothe, S., Jääskeläinen, E., Sedlar, J., Benas, N., van Zadelhoff, G.-
J., Schlundt, C., Stein, D., Finkensieper, S., Håkansson, N., and Hollmann, R.: CLARA-A2: the second edition of the CM SAF cloud and radiation data record from 34 years of global AVHRR data, Atmos. Chem. Phys., 17, 5809-5828, https://doi.org/10.5194/acp-17-5809-2017, 2017.

Kay, J. E. and Gettelman, A.: Cloud influence on and response to seasonal Arctic sea ice loss, J. Geophys. Res., 114, D18204, https://doi.org/10.1029/2009JD011773, 2009.

Kay, J. E. and L'Ecuyer, T.: Observational constraints on Arctic Ocean clouds and radiative fluxes during the early 21st century, J. Geophys. Res.-Atmos., 118, 7219-7236, https://doi.org/10.1002/jgrd.50489, 2013.

Koren, I., Oreopoulos, L., Feingold, G., Remer, L. A., and Altaratz, O.: How small is a small cloud?, Atmos. Chem. Phys., 8, 38553864, https://doi.org/10.5194/acp-8-3855-2008, 2008.

Martins, E., Noel, V., and Chepfer, H.: Properties of cirrus and subvisible cirrus from nighttime Cloud-Aerosol Lidar with Orthogonal Polarization (CALIOP), related to atmospheric dynamics and water vapor, J. Geophys. Res., 116, D02208, https://doi.org/10.1029/2010JD014519, 2011.

Merchant, C. J., Paul, F., Popp, T., Ablain, M., Bontemps, S., Defourny, P., Hollmann, R., Lavergne, T., Laeng, A., de Leeuw, G., Mittaz, J., Poulsen, C., Povey, A. C., Reuter, M., Sathyendranath, S., Sandven, S., Sofieva, V. F., and Wagner, W.: Uncertainty information in climate data records from Earth observation, Earth Syst. Sci. Data, 9, 511-527, https://doi.org/10.5194/essd-9-5112017, 2017.

Ohring, G., Wielicki, B., Spencer, R., Emery, B., and Datla, R.: Satellite instrument calibration for measuring global climate change, B. Am. Meteorol. Soc., 86, 1303-1313, https://doi.org/10.1175/BAMS-86-9-1303, 2005.

Rossow, W. B. and Schiffer, R. A.: Advances in understanding clouds from ISCCP, B. Am. Meteorol. Soc., 80, 2261-2287, https://doi.org/10.1175/15200477(1999)080<2261:AIUCFI>2.0.CO;2, 1999.

Sassen, K. and Cho, B. S.: Subvisual-Thin Cirrus Lidar Dataset for Satellite Verification and Climatological Research, J. Appl. Meteorol., 31, 1275-1285. https://doi.org/10.1175/15200450(1992)031<3C1275:STCLDF>3E2.0.CO;2, 1992.

Saunders, R. W.: An automated scheme for the removal of cloud contamination from AVHRR radiances over western Europe, Int. J. Remote Sens., 7, 867-886, https://doi.org/10.1080/01431168608948896, 1986.

Saunders, R. W. and Grey, D. E.: Interesting cloud features seen by NOAA-6 3.7 $\mu \mathrm{m}$ images, Meteorol. Mag., 114, p. 211, 1985.

Schulz, J., Albert, P., Behr, H.-D., Caprion, D., Deneke, H., Dewitte, S., Dürr, B., Fuchs, P., Gratzki, A., Hechler, P., Hollmann, R., Johnston, S., Karlsson, K.-G., Manninen, T., Müller, R., Reuter, M., Riihelä, A., Roebeling, R., Selbach, N., Tetzlaff, A., Thomas, W., Werscheck, M., Wolters, E., and Zelenka, A.: Operational climate monitoring from space: the EUMETSAT Satellite Application Facility on Climate Monitoring (CM-SAF), Atmos. Chem. Phys., 9, 1687-1709, https://doi.org/10.5194/acp-9-1687-2009, 2009.

Stengel, M., Stapelberg, S., Sus, O., Schlundt, C., Poulsen, C., Thomas, G., Christensen, M., Carbajal Henken, C., Preusker, R., Fischer, J., Devasthale, A., Willén, U., Karlsson, K.-G., McGarragh, G. R., Proud, S., Povey, A. C., Grainger, R. G., Meirink, J. F., Feofilov, A., Bennartz, R., Bojanowski, J. S., and Hollmann, 
R.: Cloud property datasets retrieved from AVHRR, MODIS, AATSR and MERIS in the framework of the Cloud_cci project, Earth Syst. Sci. Data, 9, 881-904, https://doi.org/10.5194/essd9-881-2017, 2017.

Stephens, G. L., Vane, D. G., Boain, R. J., Mace, G. G., Sassen, K., Wang, Z., Illingworth, A. J., O'Connor, E. J., Rossow, W. B., Durden, S. L., Miller, S. D., Austin, R. T., Benedetti, A., Mitrescu, C., and the CloudSat Science Team: The CloudSat mission and the A-Train, B. Am. Meteorol. Soc., 83, 1771-1790, https://doi.org/10.1175/BAMS-83-12-1771, 2002.

Stocker, T. F., Qin, D., Plattner, G.-K., Alexander, L. V., Allen, S. K., Bindoff, N. L., Bréon, F.-M., Church, J. A., Cubasch, U., Emori, S., Forster, P., Friedlingstein, P., Gillett, N., Gregory, J. M., Hartmann, D. L., Jansen, E., Kirtman, B., Knutti, R., Krishna Kumar, K., Lemke, P., Marotzke, J., Masson-Delmotte, V., Meehl, G. A., Mokhov, I. I., Piao, S., Ramaswamy, V., Randall, D., Rhein, M., Rojas, M., Sabine, C., Shindell, D., Talley, L. D., Vaughan, D. G., and Xie, S.-P.: Technical Summary, in: Climate Change 2013: The Physical Science Basis. Contribution of Working Group I to the Fifth Assessment Report of the Intergovernmental Panel on Climate Change, edited by: Stocker, T. F., Qin, D., Plattner, G.-K., Tignor, M., Allen, S. K., Boschung, J., Nauels, A., Xia, Y., Bex, V., and Midgley, P. M., Cambridge University Press, Cambridge, United Kingdom and New York, NY, USA, 2013.

Stubenrauch, C. J., Rossow, W. B., Kinne, S., Ackermann, S., Cesana, G., Chepfer, H., Di Girolamo, L., Getzewich, B., Guignard, A., Heidinger, A., Maddux, B. C., Menzel, W.P., Minnis, P., Pearl, C., Platnick, S., Poulsen, C., Riedi, J., Sun-Mack, S., Walther, A., Winker, D., Zeng, S., and Zhao, G.: Assessment of global cloud datasets from satellites, B. Am. Meteorol. Soc., 2013, 1031-1049, https://doi.org/10.1175/BAMS-D12-00117.1, 2013.

Sun, B., Free, M., Yoo, H. L., Foster, M. J., Heidinger, A., and Karlsson, K.-G.: Variability and trends in US cloud cover: ISCCP, PATMOS-x and CLARA-A1 compared to homogeneityadjusted weather observations, J. Climate, 28, 4373-4389, https://doi.org/10.1175/JCLI-D-14-00805.1, 2015.
Swinbank, R. and Purser, R. J.: Fibonacci grids: A novel approach to global modelling, Q. J. Roy. Meteor. Soc., 132, 1769-1793, https://doi.org/10.1256/qj.05.227, 2006.

Vaughan, M. A., Powell, K.A., Winker, D. M., Hostetler, C. A., Kuehn, R. A., Hunt, W. H., Getzewich, B. J., Young, S. A., Liu, Z., and McGill, M.: Fully Automated Detection of Cloud and Aerosol Layers in the CALIPSO Lidar Measurements, J. Atmos. Ocean. Tech., 26, 2034-2050, https://doi.org/10.1175/2009JTECHA1228.1, 2009.

Winker, D. M., Hunt, W. H., and McGill, M. J.: Initial performance assessment of CALIOP, Geophys. Res. Lett., 34, L19803, https://doi.org/10.1029/2007GL030135, 2007.

Winker, D. M., Vaughan, M. A., Omar, A., Hu, Y., and Powell, K. A.: Overview of the CALIPSO mission and CALIOP data processing algorithms, J. Atmos. Ocean. Tech., 26, 2310-2323, https://doi.org/10.1175/2009JTECHA1281.1, 2009.

WMO 1: Recommended methods for evaluating cloud and related parameters, WWRP 2012-1, Report of the WWRP/WGNE Joint Working Group on Forecast Verification Research (JWGFVR), available at: https://www.wmo.int/pages/prog/arep/wwrp/new/ documents/WWRP_2012_1_web.pdf (last access: 31 January 2018), 2012.

WMO 2: Systematic observation requirements for satellite-based data products for climate - Supplement details to the satellitebased component of the "Implementation Plan for the Global Observing System for Climate Support of the UNFCCC (2011 update), GCOS-154, available at: https://library.wmo.int/opac/ doc_num.php?explnum_id=3710 (last access: 31 January 2018), 2011.

Wu, D. L., Baum, B. A., Choi, Y.-S., Foster, M., Karlsson, K.G., Heidinger, A., Poulsen, C., Pavolonis, M., Riedi, J., Roebeling, R., Sherwood, S., Thoss, A., and Watts, P.: Towards Global Harmonization of Derived Cloud Products, B. Am. Meteorol. Soc., 2017, 49-52, https://doi.org/10.1175/BAMS-D-16-0234.1, 2017. 